\title{
К ТЕОРИИ ИНТЕРФЕРЕНЦИОННО-ПОЛЯРИЗАЦИОННЫХ СВЕТОФИЛЬТРОВ
}

Выведены в двух вариантах простые формулы, определяющие коэффициент пропускания интерференционно-поляризационного светофильтра (без учета потерь). Эти формулы могут быть эффективно использованы для нахождения наиболее выгодных конструкций таких фильтров.

\section{Введение}

Хорошо известны описанные неоднократно в литературе интерференционно-поляризационные светофильтры, состоящие из последовательно расположенных двупреломляющих пластинок и поляризаторов. Существуют два основных типа таких фильтров. Для фильтров типа Лио (см. $\left[{ }^{1}\right]$ и подробный обзор в $\left.\left[{ }^{2}\right]\right)$ характерно наличие поляризаторов во всех промежутках между двупреломляющими пластинками. Наоборот, в фильтрах типа Шольца (см. $\left[{ }^{3-8}\right]$ ) поляризаторов в промежутках нет, а имеются только входной и выходной поляризаторы. В настоящей статье мы будем рассматривать только светофильтры типа Шольца.

Несмотря на большое число работ, посвященных теории интерференционно-поляризационных светофильтров (см., кроме предыдущих ссылок, напр., $\left.\left[{ }^{9-18}\right]\right)$, и наличие рекомендаций, направленных на улучшение их характеристик, не все еще возможности, по-видимому, в этой области исчерпаны. В настоящей статье мы установим близкую аналогию между теоретическим описанием светофильтров типа Шольца и теорией многослойных интерференционных пленок. Эта аналогия позволит нам перенести методы, хорошо разработанные в теории пленок (см. $\left.\left[{ }^{19}\right]\right)$, в теорию интерференционно-поляризационных фильтров. Отсюда мы получим новые возможности для нахождения усовершенствованных конструкций таких фильтров.

\section{Основные теоретические формулы}

Для описания поляризации света мы используем, следуя Эвансу [ $\left.{ }^{7}\right]$, формализм Джонса $\left[{ }^{20,21}\right]$. Приняв ось фильтра, т. е. направление распространения света, за ось $z$ и взяв в плоскости $x y$, параллельной двупреломляющим пластинкам, произвольно декартовы оси $x$ и $y$, будем описывать эллиптическую поляризацию монохроматической световой волны матрицей

$$
A=\left(\begin{array}{l}
A_{x} \\
A_{y}
\end{array}\right)
$$


где $A_{x}, A_{y}$ - комплексные амплитуды электрического вектора волны по осям $x$ и $y$. Пусть волна падает перпендикулярно на двупреломляющую пластинку, вырезанную таким образом, что оптическая ось лежит в ее плоскости. Расположение пластинки определяется ее азимутом $\varphi$, т. е. углом между оптической осью и осью $x$. Прохождение волны сквозь пластинку сопровождается изменением ее поляризации; по выходе из пластинки матрица поляризации волны выражается формулой

$$
A^{\prime}=L A \text {, }
$$

где

$$
L=\left(\begin{array}{rr}
\cos \varphi & -\sin \varphi \\
\sin \varphi & \cos \varphi
\end{array}\right)\left(\begin{array}{cc}
e^{i \gamma} & 0 \\
0 & e^{-i \nu}
\end{array}\right)\left(\begin{array}{rr}
\cos \varphi & \sin \varphi \\
-\sin \varphi & \cos \varphi
\end{array}\right)
$$

и

$$
\gamma=\frac{k h\left(n_{o}-n_{e}\right)}{2}
$$

$n_{o}$ и $n_{e}-$ показатели преломления обыкновенного и необыкновенного лучей, $h$ - толщина пластинки и

$$
k=\frac{2 \pi}{\lambda}
$$

волновое число ( $\lambda$ - длина волны). По поводу формул $(2)$ и $(3)$ отметим, что здесь не учитывается ни отражение на поверхностях пластинки, ни поглощение внутри нее. Отражение может быть сделано фактически ничтожным с помощью просветляющих покрытий, а поглощение приводит только (если не учитывать дихроизма) к уменьшению общей интенсивности, не влияя на поляризацию. Во всяком случае в данной статье мы будем поглощением пренебрегать.

Введем обозначения:

$$
\begin{aligned}
& \Phi(\varphi)=\left(\begin{array}{rr}
\cos \varphi & -\sin \varphi \\
\sin \varphi & \cos \varphi
\end{array}\right), \\
& \bar{\Phi}(\varphi)=\left(\begin{array}{rr}
\cos \varphi & \sin \varphi \\
\sin \varphi & -\cos \varphi
\end{array}\right),
\end{aligned}
$$

и

$$
M(\gamma)=\left(\begin{array}{cc}
e^{i \gamma} & 0 \\
0 & e^{-i \gamma}
\end{array}\right)
$$

отметив, что матрицы Ф и $\bar{\Phi}$ удовлетворяют соотношениям:

$$
\begin{aligned}
& \Phi\left(\varphi_{2}\right) \Phi\left(\varphi_{1}\right)=\Phi\left(\varphi_{2}+\varphi_{1}\right), \\
& \bar{\Phi}\left(\varphi_{2}\right) \bar{\Phi}\left(\varphi_{1}\right)=\Phi\left(\varphi_{2}-\varphi_{1}\right), \\
& \Phi\left(\varphi_{2}\right) \bar{\Phi}\left(\varphi_{1}\right)=\bar{\Phi}\left(\varphi_{2}+\varphi_{1}\right), \\
& \bar{\Phi}\left(\varphi_{2}\right) \Phi\left(\varphi_{1}\right)=\bar{\Phi}\left(\varphi_{2}-\varphi_{1}\right)
\end{aligned}
$$

(ср. аналогичные формулы (3.23) в $\left.\left[{ }^{19}\right]\right)$. Тогда формулу (3) можно переписать в виде

$$
L=\Phi(\varphi) M(\gamma) \Phi(-\varphi)
$$


перемножая матрицы, находим другое выражение матрицы $L$ :

$$
L=\cos \gamma \cdot E+i \sin \gamma \cdot \bar{\Phi}(2 \varphi),
$$

где $E-$ единичная матрица.

Из формулы (2) вытекает, что если свет проходит последовательно сквозь $N$ двупреломляющих пластинок, перенумерованных индексами $1,2, \ldots, N$ (в направлении распространения света), то поляризация изменяется согласно формуле

$$
A^{\prime}=F A,
$$

где

$$
F=L_{N} L_{N-1} \ldots L_{1}
$$

Формулы (10)-(13) являются основными формулами теории интерференционно-поляризационных светофильтров типа Шольца.

\section{Аналогия с теорией интерференционных пленок}

Формулы (10)-(13) обнаруживают близкое сходство с соответствующими формулами теории многослойных оптических пленок (см. $\left[{ }^{19}\right]$, c. 33). Разница состоит в том, что вместо матрицы поляризации в теории пленок фигурирует амплитудная матрица, элементами которой являются амплитуды прямой и обратной волн. Матрица, преобразующая амплитудную матрицу, выражается там подобно матрице $F$ в формуле (13), а матрицы $L$ имеют вид, подобный формулам (10) и (11). Разница состоит лишь в том, что вместо матриц $Ф$ и $\bar{\Phi}$ в эти формулы входят там другие матрицы, элементы которых являются гиперболическими функциями от аргументов, связанных с показателями преломления слоев. Элементы матриц Ф и $\bar{\Phi}$ являются, аналогично, тригонометрическими функциями от (удвоенных) азимутов пластинок. Физический смысл, конечно, иной, но важно то, что соотношения (9) имеют совершенно одинаковый вид в обоих случаях. Именно это обстоятельство и делает обе теории математически вполне изоморфными, позволяя применить в теории интерференционно-поляризационных фильтров хорошо разработанные в теории интерференционных пленок методы и приемы.

\section{Преобразование основных формул}

Для преобразования матрицы $F$ имеются два основных способа (ср. $\left.\left[{ }^{19}\right], \S 8\right)$.

Во-первых, перемножая в (13) матрицы $L_{1}, L_{2}, \ldots, L_{N}$, взятые в виде (11), приходим, с учетом второй и четвертой формул (9), к выражению

$$
\begin{gathered}
F=\sum i^{s} \sin \gamma_{k_{1}} \sin \gamma_{k_{2}} \ldots \sin \gamma_{k_{s}} \cos \gamma_{l_{1}} \cos \gamma_{l_{2}} \ldots \cos \gamma_{l_{N-s}} \times \\
\times \stackrel{(-)^{s}}{\Phi}\left(2 \varphi_{k_{s}}-2 \varphi_{k_{s-1}}+-\ldots-(-1)^{s} \cdot 2 \varphi_{k_{1}}\right) .
\end{gathered}
$$

Сумма берется здесь по всем $2^{N}$ комбинациям разбиения индексов $1,2, \ldots, N$ на две группы: $k_{1}, k_{2}, \ldots, k_{s}$ и $l_{1}, l_{2}, \ldots, l_{N-s}$, причем $s=0,1, \ldots, N$ и $k_{1}<k_{2}<\ldots<k_{s} ; \stackrel{(-)^{s}}{\Phi}$ означает $\Phi$ при $s$ четном и $\bar{\Phi}$ при $s$ нечетном. Принимая во внимание формулы (6) и (7), мы можем также написать отдельные формулы для элементов матрицы $F$ : 


$$
\begin{aligned}
& F_{11}=F_{22}^{*}=\Sigma i^{s} \sin \gamma_{k_{1}} \sin \gamma_{k_{2}} \ldots \sin \gamma_{k_{z}} \cos \gamma_{l_{1}} \cos \gamma_{l_{2}} \ldots \cos \gamma_{l_{-s}} \times \\
& X \cos \left(2 \varphi_{k_{s}}-2 \varphi_{k_{s-1}}+-\ldots-(-1)^{s} \cdot 2 \varphi_{k_{1}}\right)
\end{aligned}
$$

и

$$
\begin{aligned}
F_{21}=-F_{12}^{*}= & \sum i^{s} \sin \gamma_{k_{1}} \sin \gamma_{k_{2}} \ldots \sin \gamma_{k_{s}} \cos \gamma_{l_{1}} \cos \gamma_{2} \ldots \cos \gamma_{l_{N-s}} \times \\
& \times \sin \left(2 \varphi_{k_{s}}-2 \varphi_{k_{s-1}}+-\ldots-(-1)^{s} \cdot 2 \varphi_{k_{1}}\right) .
\end{aligned}
$$

Формулы (14)-(16) аналогичны формулам (3.33)-(3.35) в [ $\left.{ }^{19}\right]$.

Второй способ требует введения матриц $\bar{M}(\gamma)$ согласно формуле

$$
\bar{M}(\gamma)=\left(\begin{array}{cc}
0 & e^{i \gamma} \\
e^{-i \gamma} & 0
\end{array}\right),
$$

причем эти матрицы вместе с матрицами $M(\gamma)$ удовлетворяют соотношениям в точности того же вида, что и матрицы $Ф$ и $\bar{\Phi}$ (см. (9)), а нменно:

$$
\begin{aligned}
& M\left(\gamma_{2}\right) M\left(\gamma_{1}\right)=M\left(\gamma_{2}+\gamma_{1}\right), \\
& \bar{M}\left(\gamma_{2}\right) \bar{M}\left(\gamma_{1}\right)=M\left(\gamma_{2}-\gamma_{1}\right), \\
& M\left(\gamma_{2}\right) \bar{M}\left(\gamma_{1}\right)=\bar{M}\left(\gamma_{2}+\gamma_{1}\right), \\
& \bar{M}\left(\gamma_{2}\right) M\left(\gamma_{1}\right)=\bar{M}\left(\gamma_{2}-\gamma_{1}\right) .
\end{aligned}
$$

Теперь перепишем формулу (13), имея в виду выражение (10) для матриц $L$ и первые формулы $(9)$ и (18), следующим образом:

$$
\begin{gathered}
F=M\left(\gamma_{N-1}-\gamma_{N-1}+\ldots+\gamma_{1}\right)\left[M\left(-\gamma_{N}-\gamma_{N-1}-\ldots-\gamma_{1}\right) \Phi\left(-\varphi_{N+1, N}\right) \times\right. \\
\left.\times M\left(\gamma_{N}+\gamma_{N-1}+\ldots+\gamma_{1}\right)\right]\left[M\left(-\gamma_{N-1}-\gamma_{N-2}-\ldots-\gamma_{1}\right) \Phi\left(-\varphi_{N, N-1}\right) \times\right. \\
\left.\times M\left(\gamma_{N-1}+\gamma_{N-2}+\ldots+\gamma_{1}\right)\right] \ldots\left[M\left(-\gamma_{1}\right) \Phi\left(-\varphi_{21}\right) M\left(\gamma_{1}\right)\right] \times \\
\times\left[M(0) \Phi\left(-\varphi_{10}\right) M(0)\right],
\end{gathered}
$$

где

$$
\varphi_{k+1, k}=\varphi_{k+1}-\varphi_{k}
$$

и

$$
\varphi_{0}=\varphi_{N+1}=0 .
$$

Далее перемножим матрицы в квадратных скобках. Результат таков:

$$
M(-\gamma) \Phi(-\varphi) M(\gamma)=\cos \varphi \cdot E+\sin \varphi \cdot \sigma_{3} \bar{M}(-2 \gamma),
$$

где

$$
\sigma_{3}=\left(\begin{array}{rr}
1 & 0 \\
0 & -1
\end{array}\right)
$$

- третья матрица Паули. Обозначим

$$
\cos \varphi_{k+1, k} \cdot E+\sin \varphi_{k+1, k} \cdot \sigma_{3} \bar{M}\left(-2 \gamma_{k}-2 \gamma_{k-1}-\ldots-2 \gamma_{1}\right)=Q_{k} .
$$

Тогда

$$
F=M\left(\gamma_{N}+\gamma_{N-1}+\ldots+\gamma_{1}\right) Q_{N} Q_{N-1} \ldots Q_{0} .
$$

Остается перемножить все матрицы в этом произведении. Учитывая 
формулы (18), а также тот факт, что матрицы $M$ и $\sigma_{3}$ коммутируют, а матрицы $\bar{M}$ и $\sigma_{3}$ антикоммутируют, находим

$$
\begin{gathered}
F=\sum_{s=0,1, \ldots, N+1}(-1)^{[s, 2]} \sigma_{3}^{s} \cdot \mathrm{X} \varphi_{N+1, N} \mathrm{X} \varphi_{N, N-1} \ldots \mathrm{X} \varphi_{10} X \\
\times \stackrel{(-)}{M}\left( \pm \gamma_{N} \pm \gamma_{N-1} \pm \ldots \pm \gamma_{1}\right) .
\end{gathered}
$$

В этой формуле $\mathrm{x}$ означает $\sin$ или cos. Сумма берется по всем $2^{N+1}$ комбинациям распределения всех х на синусы и косинусы; $s$ есть число синусов в данном члене суммы; $\stackrel{(-)}{M}^{s}$ есть $M$ или $\bar{M}$ при четном или нечетном $s$ соответственно; [ $s / 2$ ] есть целая часть от $s / 2 ;$ знаки аргумента $(-)^{n}$

$M$ определяются правилом: знак первого члена есть плюс, если $x_{\varphi_{N+1, N}}$ есть косинус, и минус, если х $\varphi_{N+1, N}$ есть синус; знаки членов $\gamma_{k+1}$ и $\gamma_{k}$ одинаковы, если х $\varphi_{k+1, k}$ есть косинус, и различны, если $\mathrm{x} \varphi_{k+1, k}$ есть синус.

По сравнению с аналогичной формулой в теорий интерференционных пленок (см. формулу (3.37) в [19]), формула (26) внешне несколько сложнее, так как содержит под знаком суммы добавочный множитель $(-1)^{[s / 2]} \sigma_{3}^{s}$. Однако на практике этот множитель учитывается очень легко, так как он равен $i^{s} E$ при $s$ четном и $i^{s-1} \sigma_{3}$ при $s$ нечетном.

Элементы матрицы $F$ выражаются согласно формуле (26) следующим образом:

$F_{11}=F_{22}^{*}=\sum_{s=0,2, \ldots} i^{s} \times \varphi_{N+1, N} \times \varphi_{N, N-1} \ldots \times \varphi_{10} \exp \left[i\left( \pm \gamma_{N} \pm \gamma_{N-1} \pm \ldots \pm \gamma_{1}\right)\right]$

и

$$
\begin{gathered}
F_{21}=-F_{12}^{*}=\sum_{s=1,3, \ldots} i^{s+1} \times \varphi_{N+1, N} \times \varphi_{N, N-1} \ldots \times \varphi_{10} \times \\
\times \exp \left[-i\left( \pm \gamma_{N} \pm \gamma_{N-1} \pm \ldots \pm \gamma_{1}\right)\right] .
\end{gathered}
$$

Эти формулы соответствуют формулам (3.38) и (3.39) в [ $\left.{ }^{19}\right]$.

\section{Заключение}

Если азимуты входного и выходного поляризаторов одинаковы и равны $0^{\circ}$ или $90^{\circ}$, то амплитуда прошедшего сквозь фильтр света равна, соответственно, $F_{11}$ или $F_{11}^{*}$ (если принять амплитуду света, прошедшего сквозь входной поляризатор, равной единице). Если же азимут входного поляризатора равен $0^{\circ}$, а выходного $90^{\circ}$, или наоборот, то амплитуда прошедшего сквозь фильтр света равна, соответственно, $F_{21}$ или $-F_{21}^{*}$. Интенсивность прошедшего света равна, следовательно, в этих двух случаях

или

$$
I_{N}=F_{11} F_{11}^{*}
$$

$$
I_{N}=F_{21} F_{21}^{*}
$$

В простейшем случае толщины всех двупреломляющих пластинок равны, т. е.

$$
\gamma_{1}=\gamma_{2}=\ldots=\gamma_{N} \equiv \gamma \text {. }
$$


Тогда, согласно формулам (15) и (16) или (27) и (28), интенсивность $I_{N}$ выражается как четный полином $2 N$-й степени от $\cos \gamma$ или $\sin \gamma$ с коэффициентами, зависящими от азимутов $\varphi_{1}, \varphi_{2}, \ldots$, $\varphi_{N}$ пластинок. От выбора азимутов зависит, следовательно, ход спектральной кривой пропускания (напомним, что величина $\gamma$ пропорциональна волновому числу). Подбирая азимуты надлежащим образом, можно в большей или меньшей степени удовлетворить задаваемым наперед условиям. Простота наших формул (15) и (16) или (27) и (28) позволяет проводить подобные расчеты наиболее эффективным образом. Соответствующая методика вместе с иллюстрирующими ее примерами будет изложена в следующей статье.

\section{Л И ТЕРА Т У РА}

1. L y ot B., Ann. Astrophys., 7, 31 (1944). Цитируется по $\left[{ }^{2}\right]$.

2. Розенберг Г. В., Оптика тонкослойных покрытий, М., 1958, с. $526-546$

3. S olc I., Чехосл. физ. ж., 4, 53 (1954).

4. S o l c I., Чехосл. физ. ж., 4, 607 (1954).

5. S olc I., Чехосл. физ. ж., 5, 80 (1955).

6. S olc I., Чехосл. физ. ж., 5, 92 (1955).

7. Evans' J. W., J. Opt. Soc. Amer., 48, 142 (1958).

8. S o l c I., J. Opt. Soc. Amer., 55, 621 (1965).

9. Evan s J. W., Appl. Opt., 2, 193 (1963).

10. Harris S. E., Ammann E. O., Chang I. C., J. Opt. Soc. Amer., 54, 1267 (1964).

11. A mmann E. O., Ch a ng I. C., J. Opt. Soc. Amer., 55, 835 (1965).

12. A mm a n n E. O., J. Opt. Soc. Amer., 56, 943 (1966).

13. A m m a n n E. O., J. Opt. Soc. Amer., 56, 952 (1966).

14. A mmann E. O., Y a r bor ough J. M., J. Opt. Soc. Amer., 56, 1746 (1966).

15. A m m a n n E. O., Y a r boroug h J. M., J. Opt. Soc. Amer., 57, 349 (1967).

16. R a alt e T. L. van, J. Opt. Soc. Amer., 57, 1217 (1967).

17. S chiffman B. M., J. Opt. Soc. Amer., 57, 1390 (1967).

18. K atz enstein J., J. Opt. Soc. Amer., 58, 1000 (1968).

19. К а рд П. Г., Анализ и синтез многослойных интерференционных пленок, Таллин, 1971.

20. Jon es R. C., J. Opt. Soc. Amer., 31, 488 (1941).

21. Ш ерк л ифф У., Поляризованный свет, М., 1965, с. $40-45$.

Тартуский государственный университет
Поступила в редакцию 30/I 1976

\section{P. KARD}

\section{INTERFERENTS-POLARISATSIOONVALGUSFILTRITE TEOORIAST}

Tuletatakse interferents-polarisatsioonvalgusfiltrite teooria põhivalemid. Maatriks $F$ (valemid (12) - (14) ja (26)) teisendab filtrile langeva valguse polarisatsioonimaatriksi $A$ filtri läbinud valguse polarisatsioonimaatriksiks $A^{\prime}$. Valemis (13) esinevad maatriksid $L$ (indeks on kaksikmurdva plaadi järjekorranumber) avalduvad valemitega (10) ja (11), kus $E$ on ühikmaatriks, maatriksid $\Phi, \bar{\Phi}$ ja $M$ on defineeritud valemitega (6)(8), $\varphi$ on kaksikmurdva plaadi asimuut ning $\gamma$ lainearvuga $k$ ja plaadi paksusega $h$ võrdeline suurus (valem (4), kus $n_{o}$ ja $n_{e}$ on hariliku ja ebahariliku kiire murdumisnäitajad). Valemis (14) $s=0,1, \ldots, N ; N$ on plaatide arv ja summa on vōetud üle indeksite $k_{1}, k_{2}, \ldots, k_{s}$ kõigi kombinatsioonide $\left(k_{1}<k_{2}<\ldots<k_{s}\right) ; \stackrel{(-)^{n}}{\Phi}$ on $\bar{\Phi}$, kui $s$ on paaritu arv, ning $\Phi$, kui $s$ on paarisarv. Valemis (26) esinevad suurused $\varphi_{k+1, k}$, $\varphi_{0}, \varphi_{N+1}$ ja maatriksid $\sigma_{3}$ ja $\bar{M}$ on defineeritud valemitega (17), (20), (21) ja (23); $\mathrm{x}$ on $\cos$ või sin, kusjuures summa on vōetud, tüle kõigi võimalike koosinuste ja siinuste kombinatsioonide; $s$ on siinuste arv mingis summa liikmes; $M$ on $\bar{M}$, kui $s$ on paaritu $\underset{(-)^{*}}{\operatorname{arv}}$, ning $M$, kui $s$ on paarisarv; kui $\mathrm{x} \varphi_{N+1, N}$ on $\cos \varphi_{N+1, N}$ või $\sin \varphi_{N+1, N}$, siis $\stackrel{(-)^{s}}{M}$ argumendi avaldises on esimene liige vastavalt $+\gamma_{N}$ vỗi $-\gamma_{N} ;$ kui $x \varphi_{k+1, k}$ on $\cos \varphi_{k+1, A}$ vổi $\sin \varphi_{k+1, k}$, siis on liikmete $\gamma_{k+1}$ ja $\gamma_{k}$ märgid vastavalt kas ühesugused vōi erinevad. 


\section{P. KARD}

\section{ON THE THEORY OF BIREFRINGENT CHAIN LIGHT-FILTERS}

New fundamental formulae describing the birefringent chain light-filters of Solc type are derived. The matrix $F$ (see formulae $(12)-(14)$ and $(26)$ ) transforms the polarization matrix $A$ of the entering light into the polarization matrix $A^{\prime}$ of the emerging light. The matrices $L$ in (13), having indices of the birefringent plates, are defined by formulae (10) or (11), where $E$ denotes the $2 \times 2$ unit matrix, the matrices $\Phi, \bar{\Phi}$, and $M$ are defined by formulae $(6)-(8), \varphi$ is the azimuth of the plate and $\gamma$ is defined by formula (4), where $k$ is the wave-number, $h$ is the thickness of the plate and $n_{o}, n_{e}$ are its refractive indices. In formula (14) $s=0,1, \ldots, N, N$ being the number of plates. Summation runs over all the combinations of indices $k_{1}, k_{2}, \ldots, k_{s}\left(k_{1}<\right.$ $<k_{2}<\ldots<k_{s}$ ); $\Phi$ is $\bar{\Phi}$ (or $\Phi$ ), when $s$ is odd (or even). The quantities $\varphi_{k+1, k}$, $\varphi_{0}, \varphi_{N+1}$ and the matrices $\sigma_{3}$ and $\bar{M}$ in formula (26) are defined by formulae (17), $(20),(21)$ and $(23)$; $x$ means cosine or sine; summation runs over all the possible combinations of cosines and sines, $s$ being the number of $\operatorname{sines;~} \stackrel{(-)^{s}}{M}$ is $\bar{M}$ (or $M$ ), when $s$ is odd (or even); the signs of the $\gamma$ 's in the argument of $M$ are as follows: $\gamma_{N}$ has +, if $\times \varphi_{N+1, N}$ is cosine, and -, if $\mathrm{x} \varphi_{N+1, N}$ is sine; $\gamma_{k+1}$ and $\gamma_{k}$ have the same signs, if $\mathrm{x} \varphi_{k+1, k}$ is cosine, and have the opposite signs if $\mathrm{X} \varphi_{k+1, k}$ is sine. 